\title{
Anticiper et simuler les dynamiques de changement pour diagnostiquer et améliorer la résilience d'un système territorial urbain
}

\author{
Anticipating and simulating the change dynamics to diagnose and \\ improve the resilience of an urban territorial system
}

\author{
Christine Voiron-Canicio ${ }^{1}$, Jérôme Dutozia ${ }^{1}$ \\ ${ }^{1}$ Université Côte d'Azur, CNRS, ESPACE, christine.voiron@unice.fr, jerome.dutozia@unice.fr
}

\begin{abstract}
RÉSUMÉ. Cette contribution traite de la résilience des villes dans une optique résolument globale. De fait, elle mobilise le concept de résilience à la fois dans une logique de gestion des perturbations de type évènementiel, dont les dynamiques spatio-temporelles opèrent dans le temps court, ainsi que dans une logique d'anticipation des futurs urbains, traitant alors de dynamiques de changement des territoires qui opèrent dans le temps long. Un exemple de diagnostic est proposé sur la spatialisation des effets dominos d'inondations et de coupures électriques dans la commune de Marseille. Dans un second temps, la résilience des villes est pensée en termes de développement des territoires et de détections de possibles tensions et de nouvelles situations à risques que pourraient faire émerger des stratégies d'aménagements, des décisions de gestion des pénuries en eau ou encore la diffusion d'innovations sur un territoire.

ABSTRACT. This contribution deals with resilient cities and explicitly takes an overall perspective. The concept of resilience is here used to examine both the event-type perturbations, which process in a relatively short time frame, and the anticipation of urban futures and territorial change dynamics, which process in a long term frame. We propose a practical exemplary of dominos effects spatialization diagnosis during flood and electric power failure in Marseille. Then, resilience of cities is linked with territorial development issues, anticipation of possible tensions and unsafe situations that may emerge due to land planning strategy, water shortages management or innovation diffusion.

MOTS-CLÉS. Résilience, Ville, Réseaux techniques, Prospective, Innovation territoriale, Risque.

KEYWORDS. Resilience, City, Technical networks, Prospective, Territorial innovation, Risk.
\end{abstract}

\section{Introduction}

À l'interface des recherches sur le risque et sur la durabilité urbaine, la résilience des villes a fait l'objet de nombreuses publications, ces dernières années. L'approche qui prédomine privilégie la résilience face au risque inondation, dans une optique plus conceptuelle qu'opérationnelle. Par ailleurs, un décalage apparaît entre les recherches menées sur ce sujet, et l'intégration de démarches résilientes dans les politiques des collectivités comme dans les services publics et privés. Une illustration en est donnée par la quasi absence du terme résilience dans les retours d'expérience qui ont suivi les deux dernières graves inondations qui ont touché les grandes aires urbaines françaises, celles d'octobre 2015, sur la Côte d'Azur, et celles de mai et juin 2016, dans les bassins moyens de la Seine et de la Loire ${ }^{1}$. De leur côté, architectes et urbanistes accordent peu d'attention à la problématique du risque, et a fortiori à la résilience urbaine (Brun, 2013). À leur décharge, il faut convenir qu'il n'existe pas de cadre formel pour l'évaluation de la résilience (Hémond, 2013) et que le concept demeure flou. Il a évolué au cours du temps et selon les approches disciplinaires, d'où un large spectre de définitions qui brouille les esprits : temps de retour à l'équilibre après un choc - définition traditionnelle; capacité d'un système à intégrer dans son fonctionnement une perturbation (risque ou crise) - définition qui prévaut aujourd'hui ; quantité de désordres qu'un écosystème peut absorber tout en conservant sa

\footnotetext{
${ }^{1}$ Le terme résilience n'est cité qu'une fois dans le document de 65 pages produit par le préfet des Alpes-Maritimes, et 4 fois sur les 100 pages du rapport du Conseil Général de l'Environnement et du Développement Durable et de I'Inspection générale de l'administration sur les inondations en lle-de-France.
} 
fonction - définition de l'écologue Holling, et largement partagée; capacité d'adaptation et d'organisation d'un système pour affronter au mieux des perturbations - définition du Commissariat Général au Développement Durable - pour ne citer que les principales. Ainsi la résilience urbaine longtemps circonscrite à l'ingénierie des infrastructures s'étend désormais au fonctionnement des territoires urbains, dans ses multiples dimensions, sociales, culturelles, historiques, et dans une moindre mesure, spatiales.

Les développements qui suivent s'inscrivent dans cette acception nouvelle de la résilience urbaine. Ils l'appréhendent sous l'angle de l'anticipation des perturbations qui pourraient affecter non seulement les réseaux techniques interdépendants mais également le système urbain, et de l'estimation des mécanismes d'adaptation, existants ou à introduire, pour améliorer la résilience générale du système.

Les deux premières sections sont consacrées aux démarches d'analyse d'un système de risques, connu, avéré ou probable, ainsi qu'à la manière de prendre en compte les interactions territoireréseaux. Ces interactions territoires-réseaux sont de natures multiples, elles amènent à considérer par exemple, la différenciation de la dépendance sociétale aux services fournis par les réseaux selon les territoires d'une ville. En effet, si une approche technique du système réseau peut convenir pour évaluer la diffusion des défaillances à partir de l'endommagement d'une ou plusieurs composantes, dès lors qu'on a la volonté de développer une approche plus holistique de l'évènement et du système de risques, tant au niveau de sa construction que de la diffusion de ses impacts, il s'avère indispensable de prendre en compte les interdépendances des territoires (espace et population) et des réseaux (infrastructure et service).

La troisième section propose une démarche consistant à rechercher des situations susceptibles de faire émerger un risque nouveau, dans un futur proche ou lointain, et d'évaluer la capacité du système territorial à le neutraliser ou l'atténuer.

Dans les deux premières sections, l'optique est de type diagnostic, dans la troisième, elle est de type veille anticipative. Les exemples en appui des présentations méthodologiques concernent deux aires urbaines méditerranéennes françaises, Marseille et Nice, soumises à divers risques naturels inondation, incendie, surcote marine - qui se conjuguent avec l'aléa défaillance électrique.

\section{Diagnostiquer le système de risques et ses interactions avec la ville}

Dans l'analyse de la résilience urbaine, les réseaux tiennent une place majeure en raison de la dépendance des services à leur égard. Les réseaux techniques fonctionnent en système. Les impacts dus à l'interruption des flux qui les relient font l'objet de recherches qui visent à parfaire les connaissances sur la vulnérabilité des réseaux face à un aléa et les effets dominos qu'il génère, et à estimer les perturbations sur la sécurité, les secours, la santé, les activités de la ville, ainsi que la capacité du système à se rétablir. Moins poussées sont les analyses des interactions territoire-réseau et des vulnérabilités qui en résultent.

Les démarches exposées ci-dessous ont comme points communs de baser l'amélioration de la résilience sur une connaissance fine des perturbations d'un système urbain liées à la survenue d'un aléa connu, et de détecter les réactions en chaîne qui découlent des interdépendances des réseaux.

\subsection{Ingénierie de la résilience urbaine et recherche-action}

La résilience des réseaux est considérée comme un préalable nécessaire, mais pas suffisant, à la résilience de la ville (Barroca et al., 2012, Lhomme, 2012; Toubin, 2014) aussi, l'ingénierie de la résilience, s'emploie-t-elle à anticiper les risques de défaillance des infrastructures, à détecter les dysfonctionnements, à évaluer les possibilités de continuité des services, et la capacité de rétablissement. Les aires urbaines soumises au risque d'inondation, et/ou touchées par des coupures 
électriques sont les terrains privilégiés pour les expérimentations de méthodes et d'outils d'aide à la décision.

Les recherches qui sont conduites dans ce domaine reposent sur :

- Une approche intégrée pour comprendre les interdépendances multidimensionnelles des réseaux et des services - électricité, hydrocarbures, gaz, eau, télécommunications - pour ne citer que les plus influents sur les autres services urbains. Dans ce cadre, la structure du système des services urbains se caractérise par un état à un temps $\mathrm{t}$, déterminé par des flux d'entrée qui sont les ressources nécessaires au fonctionnement du système et les flux de sortie, ressources nécessaires au fonctionnement d'autres systèmes. Le nombre d'intrants détermine son potentiel de défaillances, et le nombre d'extrants, son potentiel de propagation.

- L'analyse du triptyque ville-réseau-risque qui s'appuie sur la connaissance des interactions des réseaux techniques et des réseaux d'acteurs - gestionnaires, opérateurs de services, etc.

- Un diagnostic des interdépendances, plus ou moins poussé selon les niveaux spatiaux considérés. Il s'opère généralement en deux temps. Tout d'abord, un autodiagnostic sectoriel mené par chaque service, public et privé, destiné à déceler les défaillances, leurs causes et leurs conséquences; puis, le croisement des interdépendances et des défaillances lors d'ateliers collaboratifs permettant de partager cette connaissance, de hiérarchiser les problèmes au moyen d'un graphe d'interdépendances à partir duquel s'ouvre une phase de réflexion sur des pistes de solutions techniques et organisationnelles (Toubin, 2014).

L'outil de décision HAZUR ${ }^{\circledR}$ est conçu selon ce modèle. Développé par Opticits, spin-off issue de l'Université Ramon Lhull et de la ville de Barcelone, HAZUR ${ }^{\circledR}$ est à la fois une méthode et un logiciel d'analyse et gestion de la résilience, qui permet, face au risque urbain, naturel et/ou technologique, d'une part, de diagnostiquer les points critiques dans les interconnexions des services tant privés que publics, et d'autre part, de rendre la gestion de la ville plus transversale. HAZUR ${ }^{\circledR}$ a été créé en partant de la prémisse que tous les services doivent être gérés de façon intégrée. Un premier module permet de recueillir, d'analyser et de fusionner des informations diverses issues de capteurs, de bases de données, et de dires d'expert. La méthode prévoit l'organisation d'ateliers des acteurs de la ville afin d'obtenir les informations critiques sur les services, les infrastructures, leurs interdépendances, les conditions environnementales et les conséquences des impacts. Cette phase aboutit à l'élaboration du graphe des interdépendances des réseaux de services et des infrastructures. Un deuxième module permet de surveiller la ville à partir d'indicateurs clés, d'anticiper des évènements éventuels et de réagir rapidement. Il donne également la possibilité de simuler les effets des impacts sur les infrastructures et sur la temporalité de la propagation des effets dominos, d'identifier des points de rupture dans la continuité des services et le temps nécessaire pour retrouver le fonctionnement initial. Des simulations peuvent être réalisées pour détecter des effets cascade consécutifs à une option d'amélioration entraînant des changements dans les réseaux de services et d'infrastructures.

Un autre outil, le système expert DOMINO, a été développé par le Centre Risque \& Performance de Polytechnique Montréal, selon « l'approche par conséquences ». Il évalue les impacts de la perte d'une ressource d'une infrastructure sensible - appelée réseau essentiel (RE) - sur d'autres RE interdépendants. La résilience prend appui sur les notions d'acceptabilité des défaillances et de continuité d'activité. Elle est envisagée comme l'aptitude à maintenir des conditions acceptables de fonctionnement, malgré des perturbations, permettant d'assurer la sécurité des personnes et des biens. Le potentiel de résilience d'un réseau est établi sur la base d'indicateurs opérationnels renseignant sur la capacité à gérer l'imprévu, et à surveiller ce qui se passe en interne ainsi que dans l'environnement extérieur. Comme dans le modèle HAZUR ${ }^{\circledR}$, DOMINO permet de réaliser des exercices de simulation pour apprendre à appréhender la complexité des interdépendances. 


\subsection{L'insuffisante intégration de l'espace et du temps dans le diagnostic du système de risques}

Dans les applications présentées, la dimension spatiale consiste, en premier lieu, à reconstituer, au moyen d'une carte, l'étendue de l'aléa et des dommages lors d'un épisode passé (Toubin, 2014), ou bien de scénarios, dans un contexte de préparation à la gestion de crise (Robert, 2012). Dès lors que le diagnostic descend à l'échelle locale, la localisation de chaque équipement et réseau informe sur sa position relative par rapport à l'aléa, et aux perturbations induites. Cette position, associée à d'autres informations telles que les connexions avec les autres équipements, l'état de vétusté de l'infrastructure renseigne sur sa vulnérabilité propre, laquelle rejaillit sur le fonctionnement de l'ensemble.

Toutefois, la connaissance des interactions territoire-réseau reste très incomplète pour diverses raisons tenant, d'une part, aux données manquantes, incertaines, non diffusées pour cause de confidentialité, et d'autre part, à la méconnaissance de la dynamique spatio-temporelle du risque. Si les approches et modélisations systémiques des phénomènes de risques sont relativement nombreuses, les approches du système de risques et des défaillances en cascade en tant que dynamiques spatiales font globalement défaut. Cette lacune est même perçue par certains auteurs comme plus fondamentalement liée à une prise en compte biaisée de l'espace et du territoire, dans l'analyse du risque : « il y a eu méprise sur l'objet risque. La pensée sur le risque reste une pensée essentiellement externalisante. C'est-à-dire qu'elle le traite comme un objet externe aux espaces qu'il touche. Or, le processus de traduction donne à penser que le risque est littéralement ancré dans les espaces » (November, 2006). La critique d'un territoire à risque où l'espace serait restreint à un rôle de support n'est d'ailleurs pas propre à l'analyse des risques. Elle peut être étendue plus largement à la question du rôle et de la prise en compte de l'espace dans la modélisation des phénomènes géographiques complexes et en particulier, ceux à l'interface de la Nature et de la Société (Voiron, 2005).

Le couplage aléa-vulnérabilité qui sert le plus souvent de fondement à la cartographie du risque apparaît alors comme une limite dans la prise en compte de la complexité spatiale des territoires à risques, qu'elle maintient par ailleurs dans une dimension passive. Cette limite s'avère d'autant plus pénalisante dans l'analyse d'effets dominos et de réactions en chaînes initiées par le comportement perturbé des systèmes territoriaux et non directement par cette perturbation.

Une autre critique importante porte sur le fait que la définition des territoires à risques est le plus souvent construite dans un univers probabiliste qui est en fait peu adapté, dans la mesure où il postule d'une hypothèse de stationnarité ne s'observant pas dans la nature ni sur des périodes longues. Cette non-stationnarité est mise en avant dans le cas des risques naturels, avec la question du réchauffement climatique et des épisodes pluvieux extrêmes. Dans le cadre des systèmes de risques liés aux coupures électriques par exemple, l'hypothèse de stationnarité qui sous-tend un modèle probabiliste est assez peu recevable, et le caractère " émergent» de crises de grandes ampleurs associées à ces coupures, renforce ce caractère inadapté. Même si les pannes électriques à petite échelle existaient déjà il y a un demi-siècle, leurs récurrences ou leurs effets sur le fonctionnement des systèmes territoriaux ont fortement varié dans l'espace et dans le temps, et se posent en des termes différents aujourd'hui qu'ils ne se posaient en 1980 ou en 1950, en raison de la taille des réseaux mais également en raison de la dépendance sociétale accrue. D’autre part, plusieurs auteurs soulignent le caractère « hors cadres » de plusieurs évènements associés à ces défaillances des réseaux techniques. Cette absence de cadre, c'està-dire de référentiels rétrospectifs fiables sur une longue période, contribue également à rendre l'approche probabiliste peu adaptée aux systèmes de risques. Enfin, dans un système de risques ancré dans les interdépendances réseaux-territoires, l'analyse de la vulnérabilité d'un lieu n'a de sens que si elle prend en compte la vulnérabilité de l'ensemble du ou des systèmes de lieux auquel il appartient.

Pour les risques associés aux défaillances des réseaux techniques, les causes profondes qui conduisent à l'émergence d'évènements à risques de grande ampleur peuvent être organisées autour de quatre axes : la croissance des réseaux techniques et de leurs interdépendances, l'interdépendance 
spatiale produite par la structure des réseaux techniques, la dépendance sociétale aux services fournis par les réseaux, la dépendance environnementale, l'exposition à des impacts naturels et la fragilité matérielle des réseaux techniques.

Dans ce cadre, la recherche ne porte plus uniquement sur la dimension réticulaire du système électrique, mais bien sur les interdépendances réseaux - territoires et les systèmes de risques inhérents à cette interdépendance. La capacité de propagation des pannes sur des échelles spatiales importantes ne constituerait pas un risque si parallèlement la dépendance sociétale à l'électricité était très faible, ou si l'électricité était facilement substituable par une autre énergie. C'est donc la combinaison de ces phénomènes et non leur réalisation distincte qui constitue le terreau des systèmes de risques. Un intérêt de cette recherche réside dans ce parti pris : considérer le risque au niveau des défaillances des réseaux techniques mais aussi et surtout, au niveau du comportement dynamique des systèmes territoriaux soumis à une perturbation des réseaux techniques. De plus, ancrer le système de risques dans les interdépendances des réseaux et des territoires plutôt que le « centrer » uniquement sur le réseau nous paraît une perspective plus adaptée à une analyse géographique puisque « le fondement de la géographie, est d'identifier et comprendre les lieux, les réseaux qui les lient, les territoires qu'ils forment. » (R. Brunet, 1995).

Dans un contexte d'interconnexions des réseaux techniques sur de grandes distances et plus largement de fonctionnement globalisé des territoires et des réseaux, il en découle une forme de vulnérabilité qu'on peut qualifier d'ubiquiste. Si on prend l'exemple d'une ville française face au risque de coupure électrique par exemple, cette coupure pouvait être liée à une avarie sur un système fonctionnant à l'échelle régionale en 1930, à l'échelle nationale à partir de 1946 et à l'échelle européenne à l'heure actuelle. À partir de là, on comprend qu'il est tout simplement devenu impossible d'envisager l'ensemble des scénarios d'endommagement possibles pour un lieu donné. À cet égard, le retour d'expérience réalisé sur les inondations de 2016 dans les bassins de la Seine et de la Loire souligne la nécessité d'intégrer incertitude et dynamique de l'aléa, et de modifier en permanence les prévisions des $\mathrm{SCHAPI}^{2}$ pour incorporer de nouvelles informations et corriger leurs diagnostics ${ }^{3}$ (CGEDD 2017).

\section{SCReD, démarche d'analyse spatiale des systèmes de risques et de détection des espaces à enjeux}

\subsection{Articuler les propriétés territoriales : Susceptibilité, Criticité, Récupération et Dépendance}

La méthode SCReD - Susceptibilité, Criticité, Récupération et Dépendance (Dutozia, 2013) est une démarche de diagnostic axée sur les structures et les dynamiques spatiales des systèmes de risques et sur la détection des espaces à enjeux et des effets de réseaux dans les systèmes de risques. La notion d'espaces à enjeux a été privilégiée plutôt que celle de territoires vulnérables, notion à laquelle est associée une déclinaison d'enjeux, correspondant à des propriétés territoriales et réticulaires susceptibles de posséder un effet sur les systèmes de risques. Une distinction est opérée entre espaces à enjeux statiques et espaces à enjeux dynamiques. Les premiers sont identifiables avant le

\footnotetext{
${ }^{2}$ Service central d'hydrométéorologie et d'appui à la prévision des inondations

${ }^{3}$ " Le maximum atteint par la crue en un lieu, l'heure et la date, mais aussi la durée de ce maximum et la rapidité de la décrue, sont les informations dont les décideurs ont besoin. Cette prévision, qui est celle d'un hydrogramme complet et non d'une cote unique, doit pour être pertinente, être entourée d'une courbe enveloppe des incertitudes qui montre qu'à la fois le maximum, la date et la durée sont incertains, dans des proportions qui sont variables selon les événements et selon l'horizon de prévision qui est déterminant pour l'appréciation de ces incertitudes. Les décideurs oublient souvent qu'une crue n'est pas un événement qui est né à un moment donné et qu'on est en mesure de prévoir dans tout son déroulement dès son origine, mais que c'est un processus dynamique, dans lequel l'assimilation, en cours de route, des informations recueillies, est essentiel pour le voir se dessiner » CGEDD 2017, p. 27.
} 
déclenchement des catastrophes, et correspondent aux lieux renfermant des populations ou des biens vulnérables, mais également aux lieux essentiels à la gestion de crise et des secours. Les seconds sont « plus difficiles à discerner car ils n'apparaissent comme tels que dans des circonstances particulières. Leur émergence est liée, par exemple, au dysfonctionnement des relations intra-urbaines dans un contexte d'inondations » (Voiron, Olivier, 2005). L'idée d'émergence de nouveaux espaces à enjeux au cours de la crise est importante et renvoie aux questions d'effets dominos et de dynamique spatiale des systèmes de risques. Elle implique de donner aux systèmes de risques une certaine épaisseur temporelle, et à l'espace, une dimension d'agent dans le système de risques, afin de prendre en compte des impacts qui ne sont pas le résultat direct et immédiat de la perturbation mais le résultat indirect et différé du comportement perturbé du système territoire-réseau.

La démarche articule les concepts de susceptibilité, de criticité, de récupération et de dépendance (SCReD) pour envisager les systèmes de risques à l'interface de la nature, de la technique et de la société.

La susceptibilité, « composante spatiale de l'aléa » (Malet et al., 2006) se focalise sur les propriétés des espaces favorables aux déclenchements des systèmes de risques. On retrouve assez communément cette acceptation de la susceptibilité notamment dans l'analyse des mouvements de terrain (Barchi et al., 2003). La susceptibilité fait référence à l'interaction territoire - réseau, dans la dimension d'un espace matériel et statique, elle renvoie à un facteur d'exposition des composantes du système technique à des menaces et de déclenchement du système de risques à partir d'un point donné de l'espace.

La criticité, renvoie, quant à elle, au potentiel de diffusion et de déclenchement d'impacts indirects d'un sous-espace sur lequel sont implantés un ou plusieurs éléments des réseaux techniques. On raisonne ici dans la dimension d'un espace relationnel, dynamique, dans lequel les réseaux permettent aux lieux de faire système, et dessinent des trajectoires de possibles diffusions des impacts.

La récupération (Serre, 2011), est centrée sur la dimension temporelle et la notion de durée. Elle renvoie à un facteur de prolongation de la durée du système du risque, avec considération des facteurs susceptibles de prolonger la rupture de services fournis par les réseaux. L'accessibilité des composantes endommagées du système technique et des zones non alimentées par les réseaux, l'importance des moyens humains ou encore la quantité d'équipements disponibles (groupes électrogènes notamment) influent sur le potentiel de récupération pour un territoire donné.

Enfin, la notion de dépendance renvoie à la variabilité des impacts possibles de l'interruption de services d'un réseau selon les individus, les activités économiques ou encore les composantes des réseaux techniques concernées par cette coupure. Ainsi, dans certains contextes, certaines activités et certains individus faiblement dépendants vont pouvoir continuer à fonctionner et vivre de manière correcte malgré l'absence de services fournis par les réseaux alors que pour d'autres cette interruption empêche toute possibilité d'activité et peut même constituer une menace vitale. La dépendance renvoie à un facteur d'aggravation du risque. Elle s'appuie sur la connaissance d'un territoire dans sa dimension sociétale (localisation des populations vulnérables et des établissements accueillant du public, notamment) et de la dépendance différenciée des populations aux services fournis par les réseaux techniques.

Les méthodes issues de la théorie des possibilités (Zadeh, 1978), des espaces géographiques flous (Rolland May, 1987) et la gestion de l'imprécision, peuvent être utilisées comme alternative aux modèles probabilistes, en prenant en compte l'incertitude dans la délimitation des territoires du risque et dans l'analyse des systèmes de risques, dans un cadre statistique moins contraignant et sans doute plus conforme à la complexité des risques dans les territoires. 
La démarche géomatique utilisée dans SCReD correspond à un diagnostic de potentialités qui porte sur les espaces à enjeux, les possibles trajectoires de diffusion des impacts, et la réalisation de scénarios complexes mêlant vulnérabilité des réseaux et des territoires.

\subsection{L'exemple des systèmes de risques inondation-coupure électrique à Marseille}

La démarche SCReD est illustrée par une étude de cas portant sur le territoire-réseau de la commune de Marseille en contexte d'inondation. La première phase du diagnostic évalue, pour un sous-espace donné, la possibilité d'être un point de déclenchement d'une coupure électrique en raison de son exposition à l'aléa inondation. L'estimation de la susceptibilité nous a conduit à proposer plusieurs méthodes de fusions de données dont le chainage permet de construire un cadre rétrospectif et spatiale fiable, ou du moins optimisant l'usage d'informations imparfaites et limitées. Parmi ces méthodes, deux ont directement trait à la gestion de données imprécises et incertaines, soit par le biais de grilles imbriquées, soit, comme dans l'exemple présenté ici, en s'appuyant sur la morphologie des objets pour introduire une forme de variabilité supplémentaire, basée sur la distinction entre un noyau de certitude et des limites floues. On considère alors qu'une information désignant un lieu à risque est plus fiable et vraisemblable au cœur du polygone que sur ses bordures. Ainsi, dans la figure 1.a, à partir d'un tracé vectoriel désignant un lieu, on établit une bande d'incertitude, qui comprend une partie à l'intérieur du polygone et une partie à l'extérieur, l'intérieur de l'objet en rouge constitue le noyau de certitude, et la possibilité pour qu'il ait été inondé par le passé est plus grande que dans les deux bandes d'incertitude. Le noyau de certitude se voit attribuer une valeur de 1, la bande d'incertitude comprise dans les limites de l'objet une valeur de 0.8 et celle non comprise dans le polygone initial, la valeur de 0.6. Si ces valeurs restent arbitraires, elles traduisent l'idée d'un gradient dans la fiabilité de l'information, très fiable au niveau du noyau, fiable sur les marges intérieures de ce noyau et moins fiable sur les marges extérieures. Enfin, nous passons dans un mode raster dans lequel pour chaque cellule, la part de surface recouverte par les trois types d'objets est mesurée, puis multipliée par les valeurs liées à leur degré de certitude. Enfin, les résultats sont additionnés (si une cellule contient différents degrés de certitude du polygone de dommages observés). On obtient alors un indice de couverture des mailles (ICM) compris entre 0 et 1 , où la valeur 1 est attribuée si la surface de la maille est entièrement recouverte par un polygone compris dans le noyau de certitude. Appliqués aux dommages d'inondations observés dans la presse entre 1950 et 2003, dans la commune de Marseille (1.b), ce premier indice cellulaire est ensuite multiplié au nombre de postes électriques pour donner une estimation d'un niveau de possibilité de déclenchement du système de risque dans un lieu donné (1.c). 


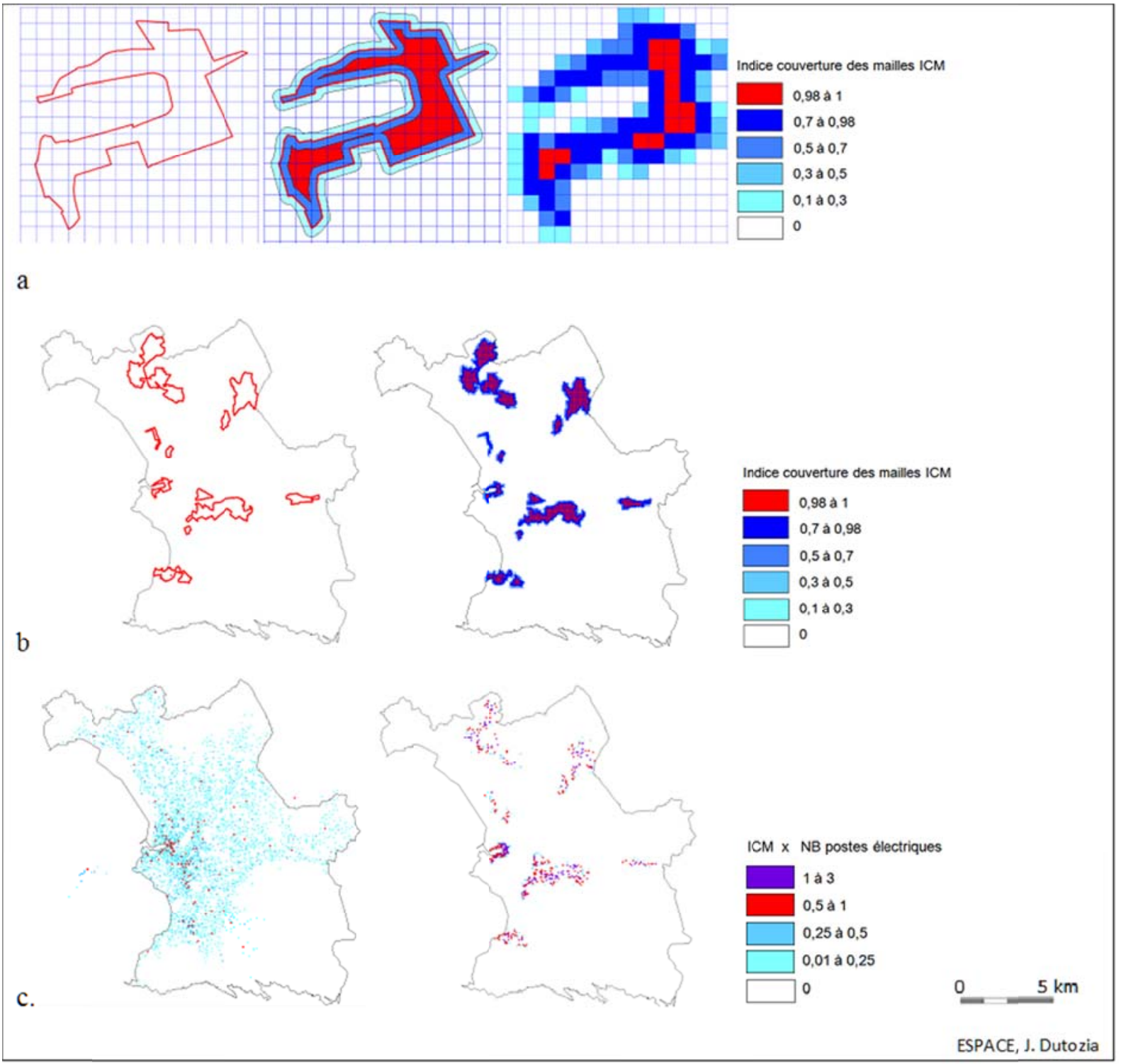

Figure I. a. Exemple de fuzzyfication d'un périmètre de zone inondable (secteur du vieux port) b. Méthodes appliquées aux zones (Iris) de la commune de Marseille citées au moins une fois dans la presse comme zones inondées entre 1951 et 2003 (pour 5 évènements de référence, 1951, 1978, 1993, 2000 et 2003)

c. Estimation de la susceptibilité par croisement avec le nombre de postes électriques.

Cette première phase renseigne sur la possibilité d'un dommage direct, et considère l'espace comme un support du système de risque dont la fonction se limite à subir l'aléa. La susceptibilité s'évalue principalement en fonction de la localisation des enjeux. À l'inverse, la seconde phase attribue à l'espace un rôle d'agent, et se focalise sur l'intensité et l'organisation des rapports d'interdépendances spatiales afin d'estimer la capacité d'un territoire à diffuser et faire émerger de nouveaux impacts en cas de perturbation. Cette propriété renvoie à la notion de criticité dans la démarche SCReD. La méthode proposée se base sur le caractère multi-niveaux du réseau électrique afin d'établir un graphe de relations verticales entre les postes électriques et l'ensemble des bâtiments de la ville (organisation hiérarchisée en trois niveaux : postes sources électriques, qui alimentent des postes de distribution électrique, qui, à leur tour, permettent l'alimentation électrique des bâtiments). Ce graphe de relations va constituer un ensemble de trajectoires potentielles du système de risques dans le sens où l'endommagement d'un poste électrique par l'aléa inondation est susceptible de provoquer un impact indirect sur les nœuds qui lui sont afférents. Sur la figure II.a, on comprend l'intérêt de ne pas considérer de manière identique l'ensemble des postes électriques compte tenu du caractère stratégique des postes sources (en noir) auxquelles sont associées plusieurs dizaines de postes de distributions. La figure II.b met en lumière la possibilité d'intégrer un degré de dépendance variable des bâtiments, en se focalisant par exemple sur l'alimentation électrique des hôpitaux, et de connaître ainsi, pour chacun des 
hôpitaux (ou autres bâtiments à enjeux de fragilité), le poste de dépendance à l'échelle des niveaux 1 et 2 de l'organisation du réseau électrique. De même, il est possible à l'échelle de chaque poste électrique, de détecter la présence de composantes territoriales dont on suppose qu'elles possèdent une forme de fragilité particulière et de dépendance électrique accrue. La détection des trajectoires susceptibles d'affecter ces cibles fragiles permet d'envisager leurs risques indirects ; par exemple, une maison de retraite peut se situer nettement en dehors d'un périmètre inondable, tout en étant dépendante d'une ou deux composantes qui sont en revanche comprises dans ce périmètre ; de fait, la maison de retraite ne fait pas partie du périmètre de risque inondation, mais fait pleinement partie du système spatial associé au système de risque inondation (Voiron-Canicio et al., 2005). Enfin, la fonction de récupération est relative à l'accessibilité routière des composantes du système technique endommagé à partir des centres de secours des exploitants des réseaux techniques. Cette accessibilité influe sur la durée de la coupure en retardant ou en rendant impossible la restauration du service fourni par le réseau. Il ne s'agit pas seulement de représenter le système territoire-réseau, mais bien d'établir un formalisme de ses interdépendances dans un système d'information géographique, qui permet ensuite de tester, à partir de requêtes spatiales, la possible réalisation de certains scénarios dans un territoire - ici, l'inondation qui conduit à l'ennoiement d'un poste source du réseau électrique et l'éventuelle rupture de l'alimentation dans un hôpital par le réseau électrique -, de savoir où cette trajectoire du système de risques pourrait se réaliser, quels types d'équipements techniques et d'établissements humains y sont intégrés.

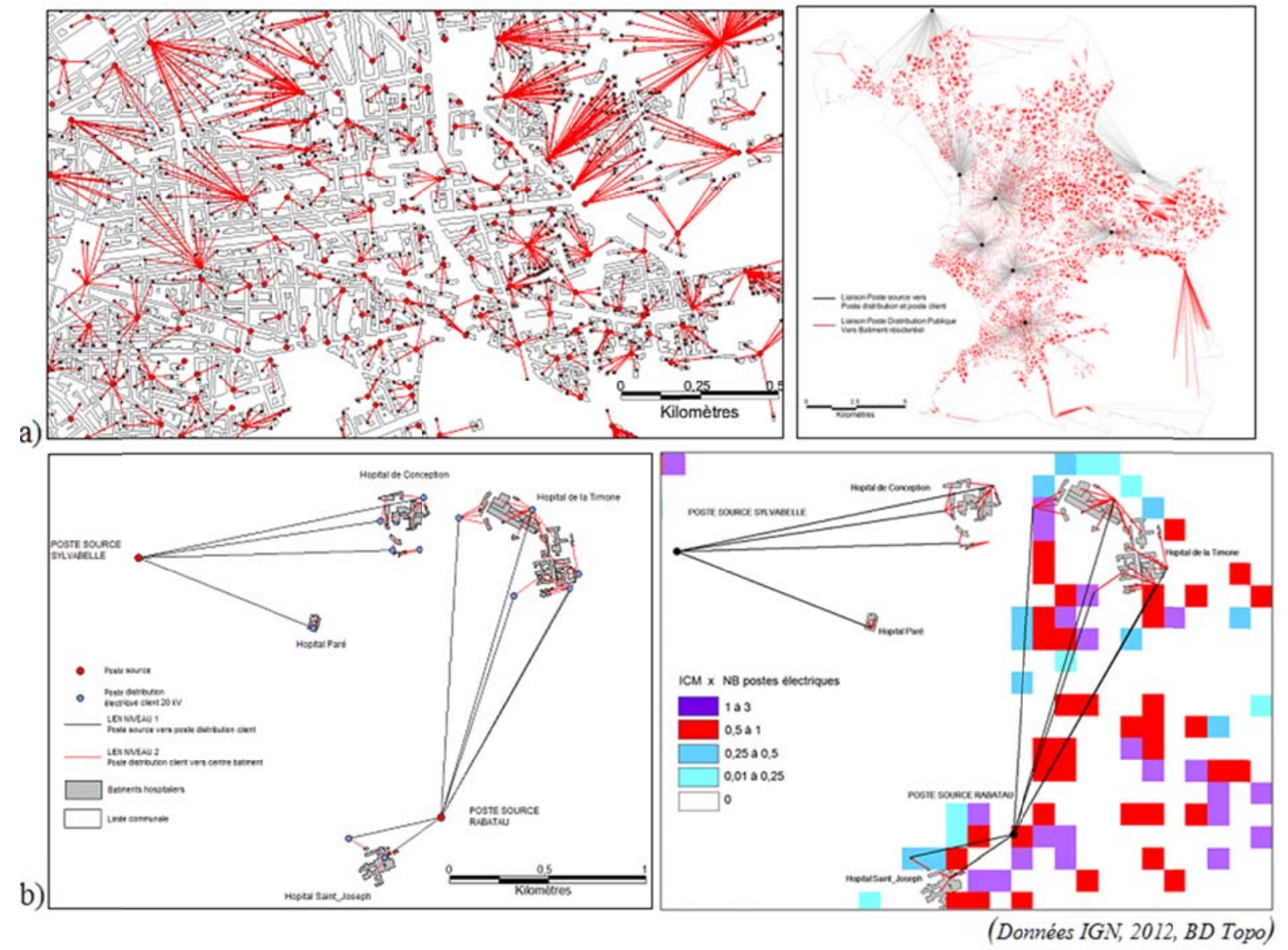

Figure II. a. Raccordement des nœuds du réseau électrique de distribution publique aux centroïdes des bâtiments résidentiels de la commune de Marseille b. Croisement susceptibilité (partie 1) avec alimentation électrique des hôpitaux (Données IGN, 2012, BD Topo)

Face à ces situations, une autre difficulté pour la compréhension et l'anticipation des systèmes de risques réside dans l'articulation entre la temporalité de la construction d'une fragilité dans le temps long, induit par le développement des territoires et des réseaux techniques, avec la temporalité plus brève et évènementielle du déclenchement des systèmes de risques induits par cette fragilité. Le 
système de risque est en ce sens « la combinaison de facteurs structurels hérités (les causes profondes de P. Blaikie et al., 1994) et de pressions et de dynamiques récentes» (Duvat, 2008). Cette combinaison sur le même espace de phénomènes se déroulant sur des temporalités différentes est un des éléments majeurs dans l'anticipation des futurs urbains et de leur résilience.

\section{Anticiper les futurs urbains par la veille anticipative}

Comprendre la dynamique spatio-temporelle du risque est de fait l'enjeu majeur de la résilience. La stratégie qui prévaut désormais est de préparer sur le long terme les processus d'adaptation qui favorisent les meilleures réponses sur le court terme (Lhomme, 2012). C'est également la stratégie préconisée pour la résilience générale d'un territoire «Trouver les réponses aux problèmes du quotidien avec des stratégies de réponse dans le temps long » (Villar, 2014). Cependant, ce long terme n'est pas donné, il est à explorer, à concevoir. Ce qui nécessite d'élargir le champ de vision aux futurs possibles. Dès lors, «la question de la résilience se pose sous l'angle de l'aptitude des acteurs à imaginer et anticiper les perturbations qui peuvent affecter la ville, et à prendre les dispositions pour les prévenir » (Commissariat Général au Développement Durable, 2015). Le scientifique figure parmi ces acteurs. Sa tâche est double, d'une part, appréhender les dynamiques de changement des territoires urbains, et déceler dans les combinaisons de processus, des situations porteuses de tensions tant environnementales que sociales, de risque de ruptures, mais également les solutions existantes ou à introduire pour y remédier, et d'autre part, transmettre ces analyses prospectives aux décideurs et populations concernées.

Cet exercice diffère de la prospective territoriale classique menée à l'échelle des grands territoires, qui analyse les systèmes territoriaux au regard d'un certain nombre de facteurs de changement: le vieillissement démographique, les avancées technologiques, les temporalités professionnelles, la variabilité climatique. L'exercice mené à l'échelle d'une aire urbaine ou d'une ville devient plus délicat pour deux raisons majeures. D'une part, la structure fonctionnelle et spatiale propre à chaque système local génère, à partir de ces facteurs de changement, des combinaisons d'évolutions pouvant s'éloigner sensiblement des trajectoires prévues ou pressenties au niveau global. D'autre part, les villes sont par essence des systèmes complexes qui évoluent en continu, en intégrant et combinant sur un même espace des dynamiques diverses. Certaines jouent sur le temps long, d'autres sur le temps court. La croissance de la population urbaine, l'étalement du bâti, le changement climatique, se rangent parmi les premières. Un aléa naturel ou technologique, une mesure de gestion, ou encore l'introduction d'une « innovation» telle que l'ouverture d'une ligne de tramway, correspondent aux secondes. Elles surviennent plus ou moins brutalement dans le système urbain, et bouleversent du jour au lendemain son fonctionnement. Face à ces perturbations lentes et brutales, l'ensemble du système urbain est amené à se réorganiser.

La résilience générale d'un système urbain découle de ces dynamiques continuelles, couplant des processus jouant sur des temporalités différentes, souvent antagonistes, et présentant des différences sensibles entre les quartiers d'une même aire urbaine. Evaluer cette résilience générale nécessite de ne pas se cantonner aux impacts des mesures de gestion mais d'appréhender l'auto-organisation du système territorial. Deux démarches peuvent être mobilisées pour tenter d'y parvenir.

\subsection{Simuler le fonctionnement d'un système urbain sur le long terme}

La modélisation en Dynamiques des Systèmes et les simulations associées aident à comprendre les réactions en chaîne qui se produisent sur le temps court mais également les répercussions durables sur la structure et la dynamique du système. Un exemple en est donné avec le prototype SERENICIM dont l'objectif est d'aider à anticiper les impacts du changement climatique sur le fonctionnement d'une aire urbaine méditerranéenne. (Voiron-Canicio et al., 2009). SERENICIM est un environnement de résolution de problèmes qui couple trois modèles. Un modèle systémique, pour intégrer la complexité 
dans le fonctionnement du système, un générateur de maquette virtuelle, pour visualiser spatialement des scénarios, et un système expert, pour proposer des mesures de gestion. SERENICIM a été conçu en prenant l'agglomération niçoise pour laboratoire d'expérimentation. Cette dernière est en effet un système urbain particulièrement complexe du fait des dynamiques environnementales et anthropiques qui interagissent, et des conflits d'usages entre l'immobilier, le tourisme, l'agriculture péri-urbaine et l'activité industrialo-commerciale, qui se déploient sur un espace de plus en plus raréfié. Le modèle systémique, dans sa version actuelle, simule le fonctionnement du territoire en faisant interagir les changements progressifs - pas de temps trimestriel jusqu'en 2050 - des paramètres physiques du climat local - températures, précipitations, variabilité saisonnière, évènements extrêmes - avec l'anthroposystème urbain dont la structure est constituée de six composantes : la population résidente, la fréquentation touristique, l'occupation du sol, les ressources en eau, la consommation d'eau selon le type d'habitat et le type d'agriculture. La composante énergétique est en cours d'introduction dans le système. La dynamique du territoire est appréhendée au moyen d'une modélisation en Dynamique des Systèmes - modèle stock-flux - où les interactions entre les composantes du système sont exprimées par des relations causales circulaires et des boucles de rétroaction positive ou négative, qui rendent compte de la complexité d'interactions difficilement perceptibles autrement. La résilience du système est appréhendée en analysant le comportement du système territorial en contexte de changement climatique progressif, ponctué d'épisodes de crise dus à des paroxysmes climatiques (pluie diluvienne, surcote marine, sécheresse, canicule).

Le risque à venir de pénurie d'eau est encore mal cerné dans les territoires urbains, notamment méditerranéens. Les effets de mesures qui pourraient être prises par les gestionnaires de la ville en cas de risque de pénurie d'eau ont donc fait l'objet de simulations. Des épisodes de sécheresse ont été introduits aléatoirement au cours de la période de simulation. Le modèle intègre des seuils d'alerte concernant les ressources en eau, qui déclenchent différents types de restrictions de consommation d'eau pour les usages domestiques et agricoles. Les simulations permettent alors d'évaluer l'efficacité des mesures adoptées en temps de crise, et leurs effets sur le temps long, et plus précisément, d'observer si les activités agricoles demeurent viables avec les mesures retenues. Il est ainsi apparu que de fréquentes restrictions de consommation d'eau avaient, à moyen terme, des impacts importants sur l'agriculture irriguée périurbaine, par ailleurs déjà fragilisée par la pression urbaine. L'exercice de simulation d'épisodes de sécheresse couplés aux interventions des gestionnaires de la ville a permis de révéler que ce qui semble être la meilleure réponse à la pénurie d'eau - problème qui se posera au quotidien dans le futur - n'est pas la meilleure stratégie pour la résilience générale de l'aire urbaine sur le temps long. Dans l'optique du maintien de l'agriculture urbaine, elle incite les décideurs et gestionnaires du territoire à réfléchir dès à présent, et de concert avec les agriculteurs, à une transition vers de nouveaux modes de cultures qui soient viables à court et à long termes.

\subsection{Anticiper la survenue de tensions et de vulnérabilités nouvelles}

Le concept de veille anticipative suppose de se projeter dans un futur qui n'est pas uniquement probable au regard du passé récent, mais également plausible, voire simplement possible, avec un double objectif, déceler des situations susceptibles de créer des tensions dans le fonctionnement de tout ou partie du territoire, pouvant conduire à de nouvelles vulnérabilités, et estimer la capacité de résilience générale du système territorial. Ce deuxième objectif n'est pas facile à atteindre. En revanche, explorer le futur est envisageable en adoptant une posture consistant à :

- repérer les tendances lourdes vs les signaux faibles dans les dynamiques en cours,

- élargir le champ de vision afin de percevoir les interactions global-local,

- être attentif aux phénomènes concomitants mais jouant sur des temporalités différentes, aux décalages dans le temps des effets de politiques publiques comme d'aménagements locaux, 
- rechercher les forces antagonistes en gestation ou susceptibles de survenir et de provoquer des tensions, les risques et les nouvelles vulnérabilités tant humaines que matérielles et organisationnelles qui en résulteraient,

- déceler les dynamiques différenciées dans l'espace, les lieux support d'innovations technologiques, les lieux créateurs d'innovations collectives, les espaces à enjeux (Voiron-Canicio, 2013).

- déterminer les facteurs pouvant jouer en faveur de la résilience, et leur localisation.

Pour illustrer cette démarche, le cas de l'anticipation des impacts d'une série d'innovations sur le devenir d'un territoire urbain sera examiné. Innovation est pris ici dans son acception générale: apparition d'une nouveauté, en un temps et un lieu donnés, produisant une rupture dans le cours des choses (Brunet, 1992). Dans le domaine de l'énergie électrique, l'ouverture d'une nouvelle ligne de tramway en est une, tout comme la mise en place d'un service d'auto-partage ou celle du compteur intelligent. Les impacts possibles de la diffusion de ce type d'innovation dans les aires urbaines de Marseille et de Nice, et tout particulièrement dans la seconde, méritent attention.

En effet, les tendances majeures observées sur ces territoires sont à la croissance du «tout électrique » mais avec des écarts sensibles entre les consommations des quartiers, et des courbes de charge très variables au niveau des postes source. Au registre des signaux faibles figurent la lente mais régulière progression de l'électromobilité, avec, comme corollaire, des inquiétudes liées à la gestion des appels de puissance sur le réseau.

Les politiques nationales engagées depuis le Grenelle de l'environnement pour lutter contre le réchauffement climatique et opérer la transition énergétique se sont multipliées. Les derniers textes de lois de 2016 et 2017, sur le pilotage énergétique local et l'autoconsommation électrique, qui autorisent à certaines conditions, les territoires à s'approprier leur volet énergétique, constituent une révolution puisqu'ils permettent une recomposition du paysage de l'offre et de la demande entre le global et le local, sans pour autant, que l'on soit en mesure d'en estimer précisément les retombées locales.

Par ailleurs, les effets de ces politiques sont attendus sur le temps long, néanmoins, elles sont déclinées localement par des actions qui ont vocation à produire des effets immédiats. Or, le décalage dans le temps des différentes actions et de leurs impacts sur le territoire est susceptible de générer des dysfonctionnements à court terme. Outre les problèmes de tension, consécutifs à l'injection de production décentralisée intermittente, il est à craindre des défaillances sur le réseau, liées aux effets conjugués de l'entrée en fonction prochaine de lignes de tramway à Nice, de nouvelles rames de métro à Marseille, et de véhicules électriques en auto-partage, dans un contexte de promotion du « tout électrique» domestique. Ce risque est particulièrement sérieux dans les Alpes-Maritimes où la sécurisation de l'alimentation électrique est difficilement maîtrisée. L'émergence de solutions de stockage de l'énergie, les smart grids et les systèmes énergétiques locaux qui éclosent déjà dans certaines zones spécifiques, ne seront pas en mesure, dans les toutes prochaines années, d'agir en complémentarité et de réguler le système. Toutefois, la mitigation par les ressources locales devrait, sur le long terme, favoriser l'équilibre global-local, facteur de résilience générale.

$\mathrm{Au}$ travers de cette projection dans le futur, il apparaît que les politiques emblématiques en faveur du développement durable peuvent, lorsqu'elles se traduisent par des actions sur le terrain, avoir des effets contraires aux impacts souhaités, comme par exemple, des tensions et des défaillances du réseau électrique dans les quartiers centraux qui rétroagissent sur tout le système urbain. Les effets dominos des coupures électriques pourraient alors générer des formes nouvelles de vulnérabilités, et fragiliser davantage les plus vulnérables. Ces processus contre-intuitifs sont rarement perçus, et requièrent des investigations au moyen de simulations. 


\section{Conclusion}

La résilience trouve ses fondements dans l'incertitude inhérente aux systèmes complexes auxquels se rattachent les villes, à la fois lieux de risques, de ruptures, et creuset d'innovations. «Plus les comportements seront complexes, plus ils manifesteront une souplesse adaptative à l'égard de l'environnement. Ils seront aptes à se modifier en fonction des changements externes, et notamment des aléas, des perturbations et des évènements, et seront aptes également à modifier l'environnement immédiat, à l'aménager, bref à adapter l'environnement au système vivant » (Morin, 1974).

Ainsi, la dynamique de changements est porteuse de risques et d'antidote au risque. La résilience urbaine réside dans cette dialectique et dans l'articulation du court terme et du long terme, du global et du local. Pour l'appréhender il est nécessaire de développer une double approche, reposant d'une part, sur un diagnostic du fonctionnement du territoire face à un système de risques connus, en portant l'attention aux évènements extrêmes auxquels le territoire doit se préparer, et d'autre part, sur l'anticipation du fonctionnement futur du territoire, susceptible de générer des tensions contenant les germes de risques nouveaux. Le chercheur désireux de mettre en pratique la veille anticipative dispose de méthodes et d'outils utilisés en prospective. Les plus adaptés sont indéniablement les outils qui s'inscrivent dans les " non path dependant approaches » (Houet, 2016), parmi lesquels figure la méthode SAS - Story and Simulation - de Joseph Alcamo (2008), qui combine, dans la construction de modèles à base de scénarios, de la connaissance quantitative - issue de modèles - et qualitative issue de la mise en récit du futur (Sgard, 2008; Vanier, 2010). Dans ce contexte de connaissance incertaine, la théorie des possibilités et ses outils associés, la métrique floue, notamment, s'avèrent d'une grande utilité pour la modélisation des scénarios prospectifs.

Sur le plan de l'opérationnalité, ces modèles prospectifs ne sont pas des outils d'aide à la décision. En revanche, ils fournissent une aide à la réflexion en amont d'un projet, et préalable à des choix d'investissements techniques d'adaptation, par exemple. Leur intérêt réside dans une approche transversale, multi-dimensionnelle de la problématique du risque urbain, et dans l'exploration de futurs possibles et pas seulement probables. Cette démarche est rarement adoptée à ce jour. Cependant, il convient de s'interroger sur l'acceptation, par les décideurs et gestionnaires locaux, de la veille anticipative, notamment lorsqu'elle amène à porter un regard distancié, voire iconoclaste, sur l'application locale de politiques publiques majeures, telle que la transition énergétique.

\section{Bibliographie}

Alcamo J., 2008, Environmental futures: the practice of environmental scenario analysis. Elsevier, Amsterdam, 212 p.

Barchi P., Marie S., Schomburgk S., Vinchon C., 2003, Cartographie de la susceptibilité aux mouvements de terrain de la commune de Barly (Pas de Calais), Rapport final, BRGM, 27 p.

Barroca B., Serre D., Y. Diab, 2012, « Le concept de résilience à l'épreuve du génie urbain », VertigO - la revue électronique en sciences de l'environnement [En ligne], Volume 12 Numéro 2 | septembre 2012, mis en ligne le 20 septembre 2012, consulté le 11 septembre 2014. URL : http://vertigo.revues.org/12469 ; DOI : 10.4000/vertigo.12469

Blaikie P., Cannon T., Davis I., Wisner B., 1994, At risk: natural hazards, people's vulnerability and disasters, London Routledge, $124 \mathrm{p}$.

Brun A., Gache F., 2013, « Risque inondation dans le Grand Paris : la résilience est-elle un concept opératoire ? », VertigO - la revue électronique en sciences de l'environnement [En ligne]. Regards / Terrain, mis en ligne le 31 décembre 2013, consulté le 17 mai 2017. URL : http://vertigo.revues.org/14339 ; DOI : 10.4000/vertigo.14339.

Brunet R., Ferras R., Théry H., 1992, Les mots de la Géographie, dictionnaire critique, Reclus, La Documentation Française, $470 \mathrm{p}$.

Dubois U., 2015, « La précarité énergétique en milieu urbain. Vers une analyse en termes de vulnérabilité », Les Annales de la recherche urbaine, Année 2015 Volume 110 Numéro 1 pp. 186-195

Dutozia J., 2013, Espaces à enjeux et effets de réseaux dans les systèmes de risques, Thèse de doctorat de Géographie, Université Nice Sophia Antipolis, 313 p. 
Duvat V., 2008, «Le système du risque à Saint Martin », Revue Développement durable et Territoires, dossier 11 « Les Catastrophes et Territoires », $19 \mathrm{p}$.

Guézo B., Pigeon P., 2015, «Les défis liés à la prévention des désastres dans les aires métropolitaines: exemple de Givors dans l'aire métropolitaine lyonnaise (France) », VertigO - la revue électronique en sciences de l'environnement [En ligne], Volume 14 Numéro 3 | Décembre 2014, mis en ligne le 27 avril 2015, consulté le 17 mai 2017. URL : http://vertigo.revues.org/15842 ; DOI : 10.4000/vertigo.15842

Hémond Y., 2013, Concepts et démarche d'évaluation du potentiel de résilience d'une infrastructure essentielle, Thèse de doctorat, Ecole Polytechnique de Montréal, 146 p.

Houet T., Aguejdad R., Doukari O., Battaia G., Clarke K., 2016, «Description and validation of a "non path-dependent" model for projecting contrasting urban growth futures», Cybergeo : European Journal of Geography [En ligne], document 759. http://cybergeo.revues.org/27397.

Lhomme S., 2012, Les réseaux techniques comme vecteur de propagation des risques en milieu urbain. Une contribution théorique et pratique à l'analyse de la résilience urbaine, Thèse de doctorat, Université Paris-Diderot, 365 p.

Malet J-P., Thiery Y., Maquaire O., Puissant, A., 2006, «Analyse spatiale, évaluation et cartographie du risque glissement de terrain », Revue internationale de géomatique, 2013, vol. 23, n² 2, p. 199-225

Morin E., 1974, «La complexité », Colloque sur les interrelations entre la biologie, les sciences sociales et la société, Paris, 18-22 mars 1974, SHC-74/CONF.801/4., 24 p.

November V., 2006, « Le risque comme objet géographique », Cahiers de Géographie du Québec, vol.50, n¹4, p.289296.

Rapport CGEDD n ${ }^{\circ}$ 010743-01 et IGA n 16080-R, 2017, Inondations de mai et juin 2016 dans les bassins moyens de la Seine et de la Loire - Retour d'expérience, $101 \mathrm{p}$.

Robert B., Morabito L., Cloutier I., Hémond Y., 2012, « An EWS Inspired Tool for Anticipating Domino Effects among Critical Infrastructures », 9e Annual symposium CRHNET, 12, p. 10-25.

Rolland May. C., 1987, « La théorie des sous-ensembles flous et son intérêt en géographie », L’Espace Géographique, Volume $16, \mathrm{n}^{\circ} 1$, p. $42-50$

Serre D., 2011, La ville résiliente aux inondations Méthodes et outils d'évaluation. Mémoire d'Habilitation à Diriger des Recherches, Université Paris-Est, 2011, 173 p. <tel-00777206>

Sgard A., 2008, Entre rétrospective et prospective, EspacesTemps.net, http://www.espacestemps.net/articles/entreretrospective-et-prospective/

Toubin M., 2014, Améliorer la résilience urbaine par un diagnostic collaboratif, l'exemple des services urbains parisiens face à l'inondation, Thèse de doctorat de Géographie. Université Paris-Diderot - Paris VII, 408 p.

Vanier M., 2010, «Le choix d'une prospective » in Délégation à l'aménagement du territoire et à l'Action Régionale, Territoires 2040, Paris, La documentation française, p. 27-31.

Villar C., 2014, « La résilience pour les territoires : outil opérationnel ou mot d'ordre incantatoire ? », Techni-cités $n^{\circ} 271$ -Juin 2014, p. 16-18.

Voiron-Canicio C., 2013, «Déceler les espaces à enjeux pour l'aménagement » in, Géogouvernance, utilité sociale de l'analyse spatiale. Masson-Vincent M., Dubus N. Ed. Collection Update, Editions Quae, p. 171-182.

Voiron-Canicio C., Olivier F., 2005, « Simulations et détection des espaces à enjeux dans un système urbain, en situation d'inondation », Actes du Colloque International SAGEO 2005, Avignon, 16 p.

Voiron-Canicio C., Dubus N., Loubier J.-C., Liziard S., 2009, « Evaluer les Impacts du Changement Climatique sur le Fonctionnement d'une Aire Urbaine Littorale : Outils d'Aide à la Réflexion et d'Aide à la Décision Existants ", Cinquième Symposium Mondial de la Recherche Urbaine, 28-30 Juin 2009, Marseille, France, http://hal.archivesouvertes.fr/docs/00/47/01/99/PDF/Colloque_URS_2009.pdf

Zadeh L., 1978, «Fuzzy sets as a basis for possibility», Fuzzy Sets and Systems 1-1978, p. 3-28. 


\section{Annexe figure II}
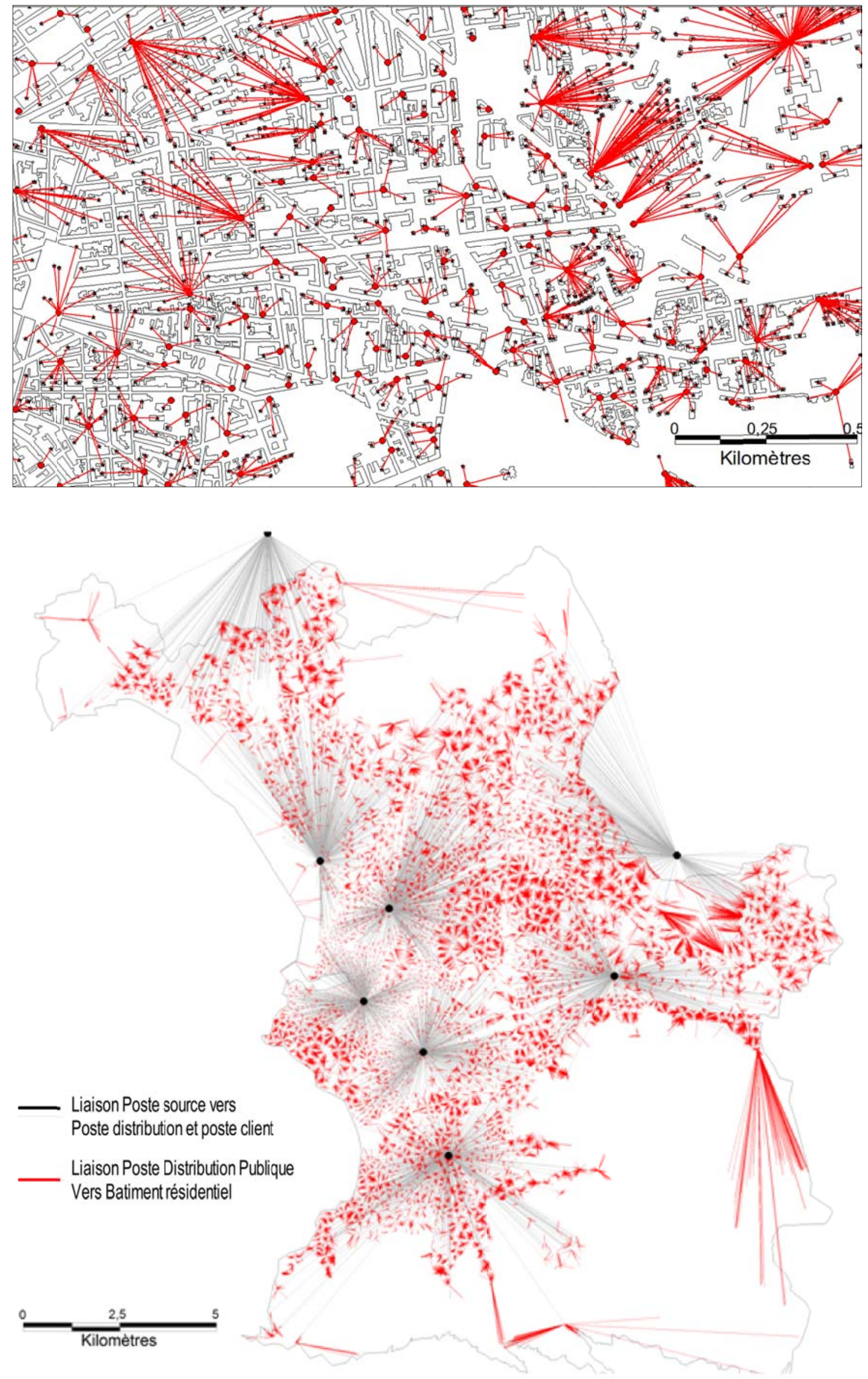

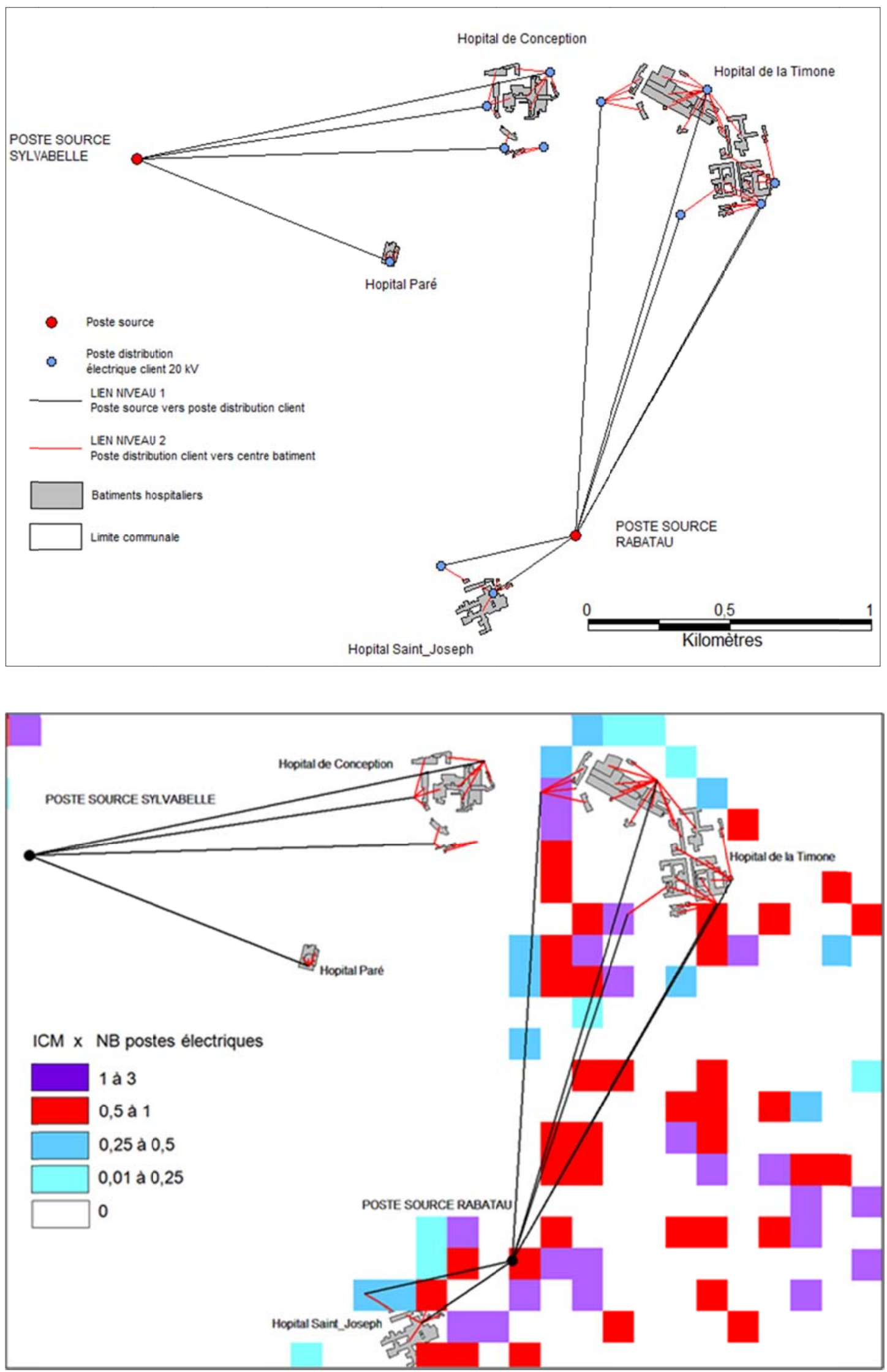